\title{
Miranda
}

Revue pluridisciplinaire du monde anglophone /

Multidisciplinary peer-reviewed journal on the English-

speaking world

5 | 2011

South and Race / Staging Mobility in the United States

\section{Facteurs de mobilité ou mobilité des facteurs (de production) ? La mise en scène urbaine du capitalisme américain}

\section{Frédéric Leriche}

\section{OpenEdition \\ Journals}

Édition électronique

URL : http://journals.openedition.org/miranda/2503

DOI : $10.4000 /$ miranda. 2503

ISSN : 2108-6559

\section{Éditeur}

Université Toulouse - Jean Jaurès

\section{Référence électronique}

Frédéric Leriche, «Facteurs de mobilité ou mobilité des facteurs (de production) ? La mise en scène urbaine du capitalisme américain », Miranda [En ligne], 5 | 2011, mis en ligne le 29 novembre 2011, consulté le 16 février 2021. URL : http://journals.openedition.org/miranda/2503 ; DOI : https://doi.org/ $10.4000 /$ miranda.2503

Ce document a été généré automatiquement le 16 février 2021.

\section{cc)}

Miranda is licensed under a Creative Commons Attribution-NonCommercial-NoDerivatives 4.0

International License. 


\title{
Facteurs de mobilité ou mobilité des facteurs (de production)? La mise en scène urbaine du capitalisme américain
}

\author{
Frédéric Leriche
}

\section{Introduction}

En économie, les facteurs de production peuvent être réduits à deux entités : le capital (ou capital constant) et le travail (ou capital variable). Les États-Unis, sur la base d'un substrat historique et culturel original - marqué durant l'essentiel du XIX $\mathrm{x}^{\mathrm{e}}$ siècle par l'expansion physique de l'espace national de référence - et comparativement aux autres grands pays industriels, sont caractérisés par une très grande mobilité spatiale de ces deux facteurs de production. À travers une analyse géographique, ce texte décrit comment la mobilité du capital se traduit dans un processus de consommation et de production d'espace, ou, plutôt et plus précisément, dans un processus de transformation et de «destruction créatrice » (pour reprendre l'idée de Schumpeter ${ }^{1}$ ) d'espace, et, parfois, de réabsorption dans le circuit économique d'espaces précédemment laissés à l'abandon. Concrètement, cette production d'espace par le capital prend la forme d'usines et d'entrepôts, d'immeubles de bureaux, mais aussi d'infrastructures diverses et de multiples espaces construits associés à l'impératif de la reproduction sociale. Dans ce processus, nous considérons que la mobilité du capital est motrice, qu'elle stimule et provoque la mobilité du travail. À ce titre, le capital, facteur de production mobile, est aussi facteur de mobilité (du travail). L'ambition première d'une telle analyse est de comprendre quelles sont les répercussions de cette mobilité des facteurs de production - et au premier chef le capital donc - sur les paysages urbains. En d'autres termes, cette analyse pose la question de savoir comment cette mobilité du capital se met en scène, pour le meilleur et pour le pire, dans les espaces 
urbains, lieux par excellence de la concentration et de l'amalgamation bien souvent frictionnelle du capital et du travail.

Dans cette perspective, précisons que l'espace n'est pas considéré ici comme la scène passive des stratégies du capital, ou, plus largement, comme le réceptacle inerte et impuissant de la vie sociale et économique. Si les recherches en économie centrées sur les firmes envisagent bien volontiers l'espace comme un simple lieu d'exercice plus ou moins neutre des stratégies du capital en mal de "localisation", nous considérons ici tout au contraire, en accord avec les analyses développées en particulier par Harvey $(2001)^{2}$, que l'espace est la pierre angulaire et l'enjeu central du capitalisme. En d'autres termes, la maîtrise de l'espace, c'est-à-dire la maîtrise des ressources, de la main d'œuvre et des marchés inscrits dans l'espace géographique, s'avère être un élément crucial des stratégies du capital, dans sa course pour l'accumulation et dans sa quête du profit. Fields (2004) par exemple, en comparant les entreprises G.F. Swift et Dell ${ }^{3}$, montre comment la question du contrôle de l'espace via les moyens de transport et de communication, permettant aux entreprises de maîtriser et de structurer les réseaux d'approvisionnement et de distribution, ainsi que, finalement, les marchés, est un élément déterminant pour asseoir la prospérité et la rentabilité de ces firmes. Comme Fields l'explique:

At the center of this comparison is a story of how transformations in business organisation are part of a general process of innovation within the firm and how the emergence of innovative enterprises reshapes the geographical territory of profit making. (1)

La mise en scène de la mobilité des facteurs de production (le capital «scénariste » et "metteur en scène " versus le travail "acteur ", voire "acté ») se traduit aux deux extrémités du processus de transformation/destruction de l'espace sous les figures emblématiques et opposées des espaces urbains où s'affiche la puissance du capital, d'un côté, et, de l'autre, des espaces où s'expriment l'échec individuel et la relégation sociale, sortes de fruits blets voire pourrissants abandonnés par le capital après usage. Cet article vise au final à montrer comment la mise en scène des succès du capital, à travers des projets urbanistiques et architecturaux d'envergure - souvent à finalités essentiellement fonctionnelles, mais aussi parfois dotés d'objectifs symboliques, communicationnels et médiatiques -, peut être comprise comme la résultante d'une stratégie d'entreprises ayant pour finalité d'accroître leur notoriété et leur expansion, et comment cette mise en scène s'accompagne - comme en "coulisse »- d'un processus parallèle de fragilisation du salariat. Ce drame, à l'échelle de l'histoire des États-Unis, se joue en cinq "actes" (au sens théâtral donc), que nous analyserons successivement à partir de trois éclairages différents (moteur technologique et industriel, inscription spatiale, mise en scène du capitalisme). Commençons néanmoins par expliciter le cadre théorique dans lequel s'inscrit notre lecture de la mobilité géographique du capital.

\section{La mobilité géographique du capital aux États-Unis : technologie, industrie, espace}

Avant d'évoquer les cinq actes de la mobilité géographique du capital, il nous faut donc expliciter le cadre théorique qui permet de définir la structuration et la temporalité de ces actes. Ce cadre insiste sur le caractère dynamique - en termes fonctionnels et 
spatiaux - de l'industrialisation, dynamique au cœur de laquelle se trouve la technologie. Soulignons au demeurant que pour analyser l'inscription de cette dynamique dans l'espace géographique, nous retenons l'échelle nationale, évacuant corrélativement de notre champ de réflexion la question de l'expansion internationale du capitalisme américain.

Certes, par bien des aspects, le capitalisme américain n'est pas très différent des autres formes de capitalisme identifiées en particulier par les économistes de l'école de la régulation (voir Boyer 2004) ${ }^{4}$. De manière générique, le fonctionnement du capitalisme (quelle qu'en soit sa forme nationale) est en effet régi par deux lois fondamentales : la baisse tendancielle des taux de profit (diminution sur le long terme des taux de profit des entreprises) et la péréquation des taux de profit (égalisation sur le long terme des taux de profit entre les branches d'activité) (Marx [1867]). À la recherche de taux de profits élevés, le capital généré par le processus de production, sous la forme de la plusvalue capitaliste, tend - pour partie - à s'investir de manière exploratoire dans de nouveaux secteurs industriels aux marges bénéficiaires plus importantes, stimulant ainsi l'essor de nouvelles technologies, mais aussi de nouveaux matériaux, de nouveaux produits, de nouveaux procédés de production, et de nouvelles entreprises. Cette dynamique exploratoire renouvelle en conséquence continuellement les bases technologiques et industrielles du processus d'accumulation. Dans cette perspective, cherchant à expliquer les cycles longs identifiés par Kondratieff [1935] $]^{5}$, Schumpeter (1942) avance la notion de « destruction créatrice » qui, selon lui :

[R]évolutionne incessamment de l'intérieur la structure économique, en détruisant continuellement ses éléments vieillis et en créant continuellement des éléments neufs. Ce processus de la Destruction Créatrice constitue la donnée fondamentale du capitalisme. (106)

Schumpeter souligne que ce processus de renouvellement n'est pas régulier et qu'il connaît, tout au contraire, des phases d'accélération liées aux vagues d'innovation technologique puis des phases de ralentissement liées à l'essoufflement de ce même cycle technologique. La thèse de Schumpeter est donc que ces cycles se développent par sauts successifs, et non pas de manière linéaire et régulière, sous la forme de " grappes technologiques ».

Cependant, comme le rappellent Storper et Walker $(1989)^{7}$, la notion de destruction créatrice renvoie à un processus qui ne se déroule pas sans tensions et sans contradictions. En effet, cette notion implique que la croissance économique, telle que définie par Schumpeter, n'est pas uniquement un processus cyclique de genèse de technologies, d'entreprises, d'industries, de méthodes de production et de produits, destiné à relancer le processus d'accumulation du capital, mais qu'elle est aussi un processus dans lequel sont mis en tension l'innovant et l'existant. La notion de destruction créatrice fait donc référence à un processus plus général qui voient s'affronter des produits nouveaux et des produits anciens (innovation de produit), des manières de travailler nouvelles et des manières de travailler anciennes (innovation de procédé), etc., mais aussi, comme le soulignent Storper et Walker, des lieux de production nouveaux et des lieux de production anciens frappés d'obsolescence.

En conséquence, l'approche géographique de la question soulevée par la notion de destruction créatrice permet de montrer, de manière complémentaire à l'approche schumpétérienne, que ce renouvellement des bases technologiques et industrielles de l'accumulation s'accompagne d'un processus d'expansion spatial du capital à la fois «consommateur» et "producteur» (ou "créateur») d'espace, ou plutôt 
«transformateur» et "destructeur » d'espace. Ce processus est particulièrement marqué dans le contexte américain, dans lequel la réserve d'espace est - par opposition au contexte européen - immense. Cette approche permet finalement de souligner à quel point l'espace est une composante vitale de la dynamique générale du capitalisme en offrant toujours des opportunités pour s'extraire des contraintes imposées par les configurations spatiales héritées des phases antérieures d'accumulation. Niveau de rémunération du salariat, rapport de force capital/travail, caractéristiques du marché du travail local, caractéristiques de la matrice industrielle locale, fiscalité, ressources passives ou construites disponibles, configuration concrète du cadre bâti et de l'espace physique, etc. sont autant de contraintes que cherche à contourner le capital (voir Harvey 1985, 2001, 2007 ; voir également Messine 1987)8.

Depuis la fin $d u x \mathrm{XVII}^{\mathrm{e}}$ siècle, le processus d'industrialisation est structuré par la succession de cinq grappes technologiques schumpétériennes (tableau 1), donnant naissance à des cycles longs dont la datation précise reste l'objet de débats chez les économistes ${ }^{9}$. Ces grappes constituent le socle fondamental de la croissance capitaliste agrégée. Elles sont également le socle technologique qui étaie les différents régimes d'accumulation qui se succèdent au cours de l'histoire du capitalisme industriel. Ou encore, comme l'expriment Storper et Walker (1989):

The expanding forces of production are the hard foundation for every regime of accumulation, on which the institutional regulation of the balance between production and consumption is constructed. (203)

Ces grappes technologiques sont, en d'autres termes, le socle sur lequel les modes de régulation des régimes d'accumulation peuvent se bâtir, à tâtons faut-il souligner, comme le montre la manière pragmatique et empirique dont $\mathrm{F}$. Roosevelt a forgé la politique du New Deal, laquelle posait les bases de l'État-Providence, dont la cohérence n'est apparue pleinement qu'a posteriori. Pour autant, les répercussions de ces grappes technologiques ne se limitent pas au système de production ou à leur capacité à générer la croissance générale de l'économie capitaliste, dans la mesure où elles ont également un impact sur les produits mis sur le marché, sur le renouvellement de la classe capitaliste, mais aussi sur la santé économique des régions et des nations intégrées les unes après les autres dans cette dynamique de l'industrialisation capitaliste.

Tableau 1. Les grappes technologiques de Schumpeter et les cycles longs : Périodisation, moteurs technologiques et industriels

\begin{tabular}{|l|l|l|l|}
\hline Cycle & Datation & $\begin{array}{l}\text { Point } \\
\text { retournement }\end{array}$ & Moteurs \\
\hline 1 & $1788-1848$ & $1815 / 1820$ & Charbon ; machine à vapeur ; coton et textile \\
\hline 2 & $1848-1896$ & 1873 & $\begin{array}{l}\text { Charbon ; acier et sidérurgie ; chemin de fer ; machine- } \\
\text { outil }\end{array}$ \\
\hline 3 & $1896-1948$ & $1918 / 1929$ & $\begin{array}{l}\text { Electricité ; industrie chimique ; industrie pétrolière ; } \\
\text { moteur à combustion ; automobile }\end{array}$ \\
\hline 4 & $\begin{array}{l}1948-1992 \\
(1998 ?)\end{array}$ & 1973 & $\begin{array}{l}\text { Pétrole ; nucléaire ; électronique ; automobile ; } \\
\text { aéronautique et espace ; pétrochimie et pharmacie }\end{array}$ \\
\hline
\end{tabular}




\begin{tabular}{|l|l|l|l|}
\hline 5 & $\begin{array}{l}1992 / 1998- \\
?\end{array}$ & $2017 ?$ & $\begin{array}{l}\text { Industries de haute technologie ; fonctions directionnelles } \\
\text { et financières ; industries culturelles }\end{array}$ \\
\hline
\end{tabular}

Source : d'après Arnold et Guy (1989)

Chacune de ces grappes technologiques (et corrélativement chaque cycle long d'expansion du capitalisme américain) est associée à une configuration géographique singulière. Cependant, la lecture de la géographie de l'industrialisation aux États-Unis est difficile dans la mesure où cette industrialisation produit une «cartographie » de l'industrie extrêmement complexe. Plusieurs paramètres viennent en effet brouiller la lecture de l'espace économique américain.

Premièrement, la carte actuelle de l'industrie aux États-Unis est le fruit de la sédimentation historique des cinq cycles longs d'expansion (décrits ci-dessous), lesquels se combinent, s'hybrident et se chevauchent. Deuxièmement, chaque grappe technologique est étroitement associée à un espace moteur initial, considéré comme l'espace central du cycle correspondant. Troisièmement, l'émergence de chaque grappe a tendance à s'inscrire dans un lieu nouveau par rapport au cycle d'expansion précédent, provoquant le déplacement du centre de gravité de l'impulsion industrielle, et provoquant l'essor successif de villes, voire de régions entières à l'échelle des ÉtatsUnis, mais aussi de nations nouvelles à l'échelle globale ${ }^{10}$; corrélativement, à chaque cycle, l'économie-monde s'élargit (Hall 1985). Quatrièmement, à mesure que se déroule un processus de maturation des secteurs industriels, les espaces centraux liés aux grappes technologiques successives tendent à déconcentrer vers des espaces périphériques les fonctions de production banale; c'est par exemple le cas de la Silicon Valley, dans le secteur de l'industrie électronique, qui « délocalise » à partir des années 1980 certaines fonctions de production dans le Sud-ouest des États-Unis, comme à Phoenix par exemple, ou en Asie du Sud-est (voir Norton et Rees 1979; Markusen 1985 ; Scott et Angel 1987). Cinquièmement, les espaces centraux d'une industrie peuvent se déplacer vers d'autres régions ou d'autres nations à l'occasion de l'apparition de nouvelles techniques ou de nouvelles technologies qui, pouvant transformer les produits pour les rendre plus attrayants sur le marché et les méthodes de production pour les rendre plus performantes, seraient mieux maitrisées par de nouveaux lieux centraux de production. C'est précisément ce qui se produit dans le cas de l'industrie cinématographique américaine qui bascule de New York à Hollywood entre 1915 et 1920 (Scott 2005; Leriche et Scott 2008). Sixièmement, des espaces dont le développement repose sur des technologies apparues lors des précédentes phases d'expansion sont susceptibles de connaître de graves difficultés, voire de s'effondrer complètement, si leurs industries ne sont pas à même d'absorber les nouvelles technologies ou si leur tissu industriel n'est pas capable d'opérer un changement sectoriel radical (à l'instar de Pittsburgh ou Detroit). Septièmement, depuis les années 1980, les investissements directs à l'étranger (IDE) en provenance d'industries de pays extérieurs (d'Europe ou d'Asie en particulier) utilisent les États-Unis dans leurs propres stratégies de développement, plaçant éventuellement les espaces américains dans une situation périphérique (à l'instar des groupes automobiles Japonais et Allemands qui ouvrent des usines de production dans le « Southern Auto Corridor»).

Le premier point évoqué à l'instant est crucial et mérite quelques éclaircissements. Chacun des cinq cycles longs d'expansion économique s'inscrit en effet dans une 
structure géographique propre ; cette expansion, à la fois technologique, industrielle et spatiale, décline ainsi les cinq actes du drame de la mise en scène urbaine du capitalisme américain.

\section{Acte I : L'industrie entre en scène}

La première grappe technologique et du coup l'ensemble des secteurs industriels moteurs du premier cycle long (ensemble dans lequel l'industrie textile est assurément un secteur clef) provoque l'essor de la " première » révolution industrielle ${ }^{11}$. Celle-ci est associée à une "révolution » des techniques et de la performance de l'agriculture, qui libère une main d'œuvre nécessaire au développement industriel urbain.

Ce premier cycle long (1788-1848) émerge dans les villes portuaires et les villes marchandes du Nord-est du pays, sur la façade atlantique donc, lieu de la première colonisation européenne en Amérique du Nord. Dans ces villes, l'industrie se développe sous la forme de districts industriels, c'est-à-dire de denses agglomérations géographiques de producteurs où l'articulation entre lieu de travail et lieu de résidence des salariés est forte; cette proximité s'impose d'elle-même dans la mesure où à cette époque, faut-il le rappeler, les moyens de transport sont encore peu développés. Dans des entreprises qui sont généralement de très petites entreprises domine la figure de l'ouvrier qualifié qui, justement parce qu'il maîtrise une large palette de compétences, bénéficie d'un certain pouvoir dans sa relation avec son employeur. Ces agglomérations sont marquées par un haut degré de désintégration du processus productif entre de très nombreuses entreprises spécialisées ${ }^{12}$. Cette organisation productive, reposant sur des économies d'échelle externes ${ }^{13}$, favorise les capacités d'innovation technique et commerciale. Ainsi, la Nouvelle-Angleterre voit-elle émerger les industries mécaniques et d'armement, mais aussi plus localement l'industrie du cuir et de la chaussure à Boston (Massachusetts), l'industrie textile à Hartford (Connecticut) ou à Lowell (Massachusetts). New York se développe grâce aux industries de l'habillement, la joaillerie, mais aussi en maitrisant les activités financières et le commerce transatlantique. Plus au Sud, le tissage du coton s'ancre à Philadelphie. Cependant, à mesure que se développe et se transforme l'industrie, de nouvelles villes s'industrialisent. Progressivement, de nouvelles villes situées plus à l'intérieur des terres comme Cincinnati, Albany ou Troy se développent grâce aux industries du textile ou du travail du fer.

Lowell fournit un bel exemple de l'architecture industrielle du XIX ${ }^{e}$ siècle, massive, sobre, fonctionnelle. Espace transformé et produit par cette première phase d'industrialisation, puis abandonné avant d'être absorbé à nouveau dans le circuit économique, la ville de Lowell est désormais mise en scène, par des capitaux publics et privés combinés, en valorisant l'histoire locale. Fondée en 1821 par quelques hommes d'affaires de Boston, la ville de Lowell compte 2500 habitants en 1826, et 33000 en 1850 ; elle est alors la seconde ville du Massachusetts derrière Boston. Qualifiée en 1846 de "Manchester de l'Amérique » par Thoreau, Lowell se développe encore dans la seconde moitié du XIX ${ }^{e}$ siècle, avant de connaitre une crise sévère au XX ${ }^{\text {ème }}$ siècle. Le renouveau n'apparaît qu'à partir des années 1970, lorsque l'État du Massachusetts fonde une université et que l'entreprise Wang installe un établissement (Mangin 2002). Désormais située dans l'orbite de la métropole de Boston, Lowell a réhabilité son patrimoine industriel. Traduction de l'intérêt naissant pour l'Heritage industry, nouveau pilier du 
développement économique local susceptible d'attirer des touristes et de revaloriser l'image de marque d'un lieu, l'usine dite "Mill Building " est dans cette perspective transformée en musée (cf. figure 1) ${ }^{14}$; dans la même veine, Lowell valorise son statut de lieu de naissance de Kerouac (en 1922), et organise depuis le milieu des années 1980 un festival folk de renom.

\section{Acte II : L'ère de la machine et des barons d'industrie}

La deuxième grappe technologique, à l'origine du deuxième cycle long, ouvre la voie à l'« ère de la machine ", dans la mesure où, dominée par le travail de l'acier, elle est associée à l'apparition des machines-outils. Celles-ci accroissent le pouvoir de l'homme sur la matière travaillée, en particulier pour la transformation de l'acier, ouvrant ainsi l'éventail des réalisations possibles et accroissant continuellement la productivité. Du coup apparaît une quantité considérable de produits nouveaux : biens d'équipements pour l'agriculture, produits liés à l'essor du transport ferroviaire (locomotive, wagon), biens intermédiaires pour la construction (poutrelles, escaliers roulants et ascenseurs), biens de consommation (bicyclette, conserves alimentaires), etc. La nature des technologies mises en œuvre et des produits mis sur le marché, requérant des masses importantes de capital et de travail, offre des conditions favorables à l'émergence des premières sociétés géantes du capitalisme américain (comme Carnegie ou Pullman). Cette deuxième grappe technologique est le support de l'accumulation capitaliste de la seconde moitié du XIX siècle. Elle stimule la croissance urbaine de villes dans lesquelles lieux de travail et lieux de vie sont, toujours, situés à proximité. Cependant, l'une des conditions essentielles rendant possible cette accumulation est le recours massif et continuel à l'immigration - en provenance d'Europe - qui a le double intérêt d'alimenter le marché du travail en main d'œuvre et d'étendre le marché de la consommation (Leriche 2009). Par ailleurs, la seconde moitié du XIX ${ }^{e}$ siècle est caractérisée par un mode de régulation articulant accumulation extensive et régulation concurrentielle (Boyer 1986) ${ }^{15}$. En d'autres termes, le rapport salarial est régi de manière concurrentielle, ouvrant la voie à des relations de classes conflictuelles (en particulier en phase «descendante " du cycle d'expansion, dans le dernier quart du $\mathrm{XIX}^{\mathrm{e}}$ siècle), comme l'illustrent les luttes parfois extrêmement violentes, à l'instar des premiers mouvements de grève à Chicago en 1886 (le $1^{\mathrm{er}}$ mai) puis à nouveau en 1894 (Pullman Strike), ou encore à Pittsburgh en 1892 (usine de Carnegie Steel à Homestead). Face à la puissance du capital incarné par les grands barons d'industrie de la fin du $\mathrm{XIX}^{\mathrm{e}}$ siècle, la classe salariale se trouve en difficulté pour négocier des niveaux de rémunération suffisamment élevés, et elle reste en marge de la sphère de la consommation. En outre, consécutivement, le rythme d'expansion est modéré et erratique.

Ce deuxième cycle long (1848-1896) correspond donc à l'« âge de la machine » et de l'acier, mais aussi à l'âge d'or du «capitalisme sauvage ». Nombre de villes de la Nouvelle-Angleterre, et de la façade Atlantique plus généralement, continuent à se développer grâce à l'industrie, comme Waltham dans la banlieue de Boston, qui connaît un essor renouvelé à partir de 1854 grâce à sa spécialisation dans la fabrication de montres et d'instruments de précision. Philadelphie voit émerger les industries chimiques et mécaniques, New York ajoute l'édition dans la palette de ses industries, Baltimore devient un site de production sidérurgique et de chantiers navals (Sparrows 
Point). La hiérarchie urbaine est cependant bouleversée par le développement de villes industrielles souvent localisées vers les Grands Lacs, dans les vallées de l'Ohio et de ses affluents (Allegheny et Monongahela) ou dans le Midwest (Akron, Buffalo, Cincinnati, Cleveland, Detroit, Saint-Louis, etc.). Au Nord des Appalaches, sur un site riche en charbon, Pittsburgh, adoptant en 1875 le procédé Bessemer ${ }^{16}$, se développe pour devenir un pôle majeur de l'industrie sidérurgique mais aussi de l'industrie du verre. Sise dans une aire de production agricole, Chicago devient un grand pôle industriel grâce à l'industrie agroalimentaire (et à ses abattoirs, comme ceux de l'entreprise G.F. Swift), à l'industrie de la machine-outil et à l'industrie du transport ferroviaire. Dans ces villes, l'activité motrice prend parfois au départ un caractère de mono-industrie marqué, comme à Minneapolis-St-Paul (minoterie) et Milwaukee (brasserie) qui se développent sur la base de la transformation de ressources locales. Ces industries et ces villes deviennent alors les moteurs d'un nouveau processus de développement régional, qui donne naissance à la désormais classique Industrial Belt autour des Grands Lacs. Dans des usines de plus en plus grandes sont expérimentées de nouvelles méthodes de production à grande échelle marquant les prémisses des développements industriels ultérieurs. Pour autant, d'autres sites industriels émergent, comme sur la côte Pacifique où San Francisco se développe en valorisant un savoir-faire acquis dans la production de matériel d'extraction minière progressivement constitué à partir de Ruée vers l'or de 1848 (Walker 1996).

Comment, finalement, le capital se met-il en scène ici ? L'usine de l'une des sociétés fondatrices et emblématiques de la brasserie à Milwaukee (Cochran 1948), le Pabst Brewery Complex, dont la finalité est productive et fonctionnelle, permet de donner un exemple de la manière dont le capital se met en scène dans l'espace urbain (cf. figure 2) ${ }^{17}$. L'usine ferme en 1997 suite aux difficultés de l'entreprise. Immense complexe de bâtiments difficiles à réutiliser sans traitement urbanistique et architectural en profondeur, sorte de verrue urbaine sise non loin de downtown Milwaukee, l'établissement industriel est racheté en 2006 par un promoteur immobilier qui y conduit une opération de redéveloppement mêlant espaces résidentiels, bureaux d'entreprises, espaces commerciaux; une partie du site (les espaces de bureau et le Visitor Center) est restaurée afin de relier le projet immobilier à l'histoire du lieu industriel. L'opération de redéveloppement n'est pas achevée en 2011. Espace produit par l'industrialisation amorcée à Milwaukee à partir du milieu du XIX ${ }^{\mathrm{e}}$ siècle, puis tombé en désuétude, le Pabst Brewery Complex est donc en cours de réinsertion dans le cycle économique, par l'intermédiaire capitalisme immobilier; celui-ci met en scène cette reconversion et le changement d'usage du site en évoquant l'héritage historique ainsi que l'attachement du porteur de projet à Milwaukee ${ }^{18}$.

\section{Acte III : L'électricité et l'automobile entrent en scène}

La troisième grappe technologique, reposant sur l'électricité, la chimie et le moteur à combustion, voit se diversifier encore la gamme des industries et des produits. Correspondant à l'ère du taylorisme, elle structure le troisième cycle long. Cette phase de l'expansion industrielle est sans doute celle qui transforme le plus spectaculairement la société américaine (et la société occidentale dans son ensemble). Un processus continuel d'innovation permet en effet de mettre sur le marché une 
incessante cascade de biens et de services nouveaux (du tramway électrique à l'automobile, en passant par l'électrification des foyers, la radio, les premiers équipements électriques du foyer, et une série quasi-infinie de produits plastiques par exemple). Dans une matrice industrielle de plus en plus complexe, les connaissances, les savoir-faire et les technologies se diffusent souvent de manière transversale et interindustrielle, en fonction des spécificités et des besoins techniques des industries; par exemple, les progrès de l'industrie chimique autorisent le développement de l'industrie photographique (grâce aux pellicules photo popularisées par Kodak vers 1900), les progrès de la maitrise de l'électricité permettent la mise au point de démarreurs automobiles (vers 1910-1915). Clef de voûte et emblème de cette phase du développement industriel, l'industrie automobile a des conséquences considérables, tant sur le mode de vie (bouleversé par le produit automobile) que sur les méthodes de production (la combinaison du taylorisme et de la chaîne d'assemblage mise au point dans les usines de Ford se diffuse dans de nouveaux secteurs industriels comme les biens d'équipement ménager). Mais l'industrie automobile - tout comme les autres secteurs industriels en expansion - a aussi des effets d'entrainement sur la croissance urbaine, car elle provoque la création d'usines parfois gigantesques ${ }^{19}$, draine de grandes quantités de travailleurs, et induit corrélativement une croissance urbaine rapide, tandis que d'immenses infrastructures routières et autoroutières sont aménagées. Au cours de la période correspondant à cette grappe technologique, le mode de régulation articule accumulation intensive sans consommation de masse et régulation toujours concurrentielle. Le rapport salarial prend alors progressivement une forme taylorienne instituant une profonde réorganisation du procès de travail; les méthodes de production définies par Taylor, l'organisation scientifique du travail (OST), sont de plus en plus systématiquement appliquées, dessaisissant progressivement les ouvriers des anciennes manufactures de leurs savoir-faire polyvalents, et donc aussi d'une partie de leur pouvoir (voir Aglietta 1997). En outre, ce rapport salarial ne modifie pas de manière importante le mode de consommation de la classe ouvrière. La croissance est relativement soutenue mais cyclique. De plus en plus performant en termes de capacités productives, grâce à la diffusion des normes tayloriennes de production, le processus d'accumulation associé trouve ses limites lors de la crise de 1929.

Tout au long de ce troisième cycle long (1896-1948), l'expansion industrielle stimule le développement urbain dans les grandes villes de l'Industrial Belt. Taylorisme et standardisation permettent d'améliorer la productivité, d'abaisser les coûts de production et de renforcer la compétitivité des entreprises. De nouveaux produits, de consommation plutôt que d'équipement, apparaissent, et les économies d'échelle autorisées par la production de séries longues permettent continuellement d'étendre le marché vers de nouvelles strates de consommateurs. Les économies d'échelle renforcent en outre la spécialisation régionale de l'industrie (Krugman et Obstfeld 1995). Les évolutions amorcées dans la phase précédente se confirment donc sous la forme du développement de centres industriels parfois très spécialisés, comme les villes emblématiques de Pittsburgh (sidérurgie) ou Detroit (automobile), parfois plus diversifiés, comme Chicago (machine-outil, mécanique). La période 1910-1930 marque «les années triomphales de Detroit » (Bergeron et Mauillari 1997) ; la population de la ville passe entre 1900 et 1930 de moins de 300000 à plus de 1,5 millions d'habitants, les entreprises locales commencent à diffuser géographiquement leur production en investissant ailleurs aux États-Unis et à l'étranger ${ }^{20}$. Les industries exploitant l'électricité (appareils électroménagers, mais aussi radio, cinéma, machines de bureau, 
téléphone, etc.) se développent à New York et alentour, les industries chimiques, pharmaceutiques et cosmétiques se développent dans les régions de New York et Philadelphie, jusqu'à Wilmington (Delaware) ${ }^{21}$. La Californie du Sud devient un pôle de développement des industries du pétrole, du cinéma, de l'aéronautique et de l'armement (Scott 1991), tandis qu'émerge en Californie l'agriculture capitaliste irriguée et ses industries associées (conditionnement agro-alimentaire) ${ }^{22}$, et que l'industrialisation périphérique conduit les producteurs de Detroit à y installer des usines, au point que Los Angeles devient alors le deuxième pôle de production automobile du pays (Soja, Morales et Wolff 1983). Si la géographie économique des États-Unis devient plus complexe, malgré tout, pour un temps, la ceinture des Grands Lacs s'impose comme le cœur industriel du pays.

Inaugurée en 1908, l'usine historique de Dearborn, usine emblématique de cette troisième phase de l'industrialisation américaine, est désormais flanquée d'un musée relevant du Henry Ford Museum, le Ford Rouge Factory Visitor Center (cf. figure 3) ${ }^{23}$, destiné à valoriser l'image de marque de l'entreprise. À l'heure où l'industrie automobile américaine traverse une crise sans précédent, Ford cherche à profiter de l'engouement $\mathrm{du}$ public pour le tourisme industriel, mettant ainsi en scène (et commercialisant au passage) son savoir-faire industriel et ses compétences techniques. Cette stratégie de communication - faire savoir le savoir-faire - n'est pas sans quelque emphase relevant d'une démarche de théâtralisation du travail de production industrielle. Ainsi, la société chargée de l'animation de ce Visitor's Center, BRC (Brand Cultural Entertainment Media \& Events), vante-t-elle les mérites de ce site en le présentant comme étant une attraction touristique majeure. Comme l'explique BRC:

Here, BRC created and produced a world-class visitor experience highlighting the people, products, and processes behind Ford Motor Company's commitment to a sustainable future for industrial manufacturing. (...) Visitors get to walk through part of the assembly plant and explore the environmentally friendly $21^{\text {st }}$-century automotive production techniques employed by Ford Motor Company ${ }^{24}$.

Si l'idée de promouvoir le tourisme industriel peut séduire, la rhétorique très «tendance » vantant les préoccupations de Ford pour l'environnement laisse quant à elle quelque peu circonspect.

\section{Acte IV : Le Fordisme, l'avènement des industries de haute technologie}

La quatrième grappe repose sur des technologies de plus en plus sophistiquées alimentant de nouvelles industries: aéronautique, électronique, nucléaire. Les industries motrices de la phase antérieure, comme l'automobile ou les biens d'équipements ménagers, n'en continuent pas moins à jouer un rôle prépondérant dans les dynamiques de l'accumulation capitaliste. Les "nouvelles" technologies liées à cette grappe sont la source de secteurs industriels puissants qui, n'ayant qu'un faible impact concurrentiel et substitutif, se surajoutent donc aux industries fordistes. L'électronique a un statut particulier; en tant que technologie transversale, elle est à même de se propager dans l'ensemble de l'industrie mais aussi de susciter une immense vague de produits de consommation nouveaux. Ce cycle long est également associé à la production d'infrastructures énormes, en particulier les aéroports, tandis que s'accentue le développement des autoroutes et que s'accélère la croissance urbaine. Les secteurs de l'aéronautique, de l'espace, de l'armement et du nucléaire, dans un 
contexte marqué par la guerre froide, connaissent une expansion rapide adossée à des dépenses fédérales importantes. Le mode de régulation correspondant à cette grappe technologique est fortement marqué par le processus d'accumulation de la phase antérieure. Ce mode de régulation (souvent dit «fordiste») combine accumulation intensive centrée sur la consommation de masse et régulation monopoliste d'État. S'il se met progressivement en place à partir des années 1930, il ne s'impose définitivement qu'après la Seconde Guerre mondiale. Le rapport salarial prend alors une forme dite " fordienne ", codifiant la progression concertée - le collective bargaining - et parallèle de la production et de la consommation. Pendant une longue période (les «Trente glorieuses »), grâce à ces dispositifs établis sur une base nationale et alors que les systèmes productifs nationaux se ferment relativement aux périodes antérieures ${ }^{25}$, la croissance est soutenue et relativement stabilisée. Ce régime d'accumulation fordiste entre en crise au cours des années 1970, en raison même des dynamiques de l'industrialisation capitaliste qui a conduit à l'apparition de produits plus adaptés aux goûts des consommateurs - se substituant donc à des produits obsolètes -, à des surinvestissements productifs dans de nombreux secteurs, et à l'émergence de concurrents aux méthodes productives plus performantes comme, en particulier, le Japon. Dès lors, l'économie des États-Unis entre dans une période de croissance ralentie et cyclique, et l'industrie nationale essuie une crise sévère et alarmante (voir Bluestone et Harrison 1982).

Ce quatrième cycle long (1948-1992/98) correspond à ce qu'il est convenu d'appeler le haut fordisme. Au cours de cette phase d'expansion, les industries de production de masse continuent à se développer dans l'Industrial Belt, qui devient alors quasiment le centre économique du monde. Investissant dans d'autres régions du pays et, de plus en plus, à l'étranger afin de prendre place et de tirer profit des marchés émergents, les entreprises de ces industries continuent à pratiquer la déconcentration spatiale de la production. Le pôle dominant pour les activités d'extraction et de raffinage du pétrole et les activités pétrochimiques se déplace vers le Texas et la Louisiane, l'agriculture capitaliste irriguée se diffuse en Arizona et en Floride. Surtout, les industries de haute technologie amorcent un développement spectaculaire géographiquement ancré dans quelques lieux: nouveaux matériaux et aéronautique à Seattle et Los Angeles, industries liées à la défense (missiles, radars, etc.) en Californie du Sud, électronique et industrie informatique au Sud de San Francisco (Silicon Valley), technologies d'information et de communication dans des lieux variés comme Boston, Dallas, Denver, Minneapolis, Phoenix, etc. (Castells 1989). Au cœur même de cette phase d'expansion fordiste émerge donc un nouveau paradigme industriel à l'origine d'une nouvelle géographie économique nationale, perceptible à travers les dynamiques démographiques des principales métropoles du pays. La Sunbelt, correspondant au Sud et à l'Ouest du pays, résulte d'une redistribution géographique du capital adossée à des dépenses publiques d'armement massives à partir des années 1950 en raison de la guerre froide ${ }^{26}$. La crise des années 1970 frappe sévèrement les industries de production de masse et les espaces associés (comme la fameuse Industrial Belt qui se transforme par endroits, comme Pittsburgh, en Rusbelt, le Nord-est Atlantique, sans oublier la Californie du Sud) ${ }^{27}$.

Entreprise emblématique du dynamisme des industries de la haute technologie, fondée en 1977 par Larry Ellison, la société Oracle symbolise le dynamisme économique et territorial de la Sunbelt (cf. figure 4) ${ }^{28}$. Grâce à une croissance rapide, elle est désormais, en termes de chiffres d'affaires, la troisième société mondiale du secteur du software 
informatique derrière Microsoft et IBM. Son siège social, situé dans la Silicon Valley (Redwood City), met en scène, via son architecture moderne et sa localisation en bordure d'un lac aménagé sur la baie de San Francisco, au détriment de l'écosystème de la baie, une conception relativement ostentatoire mais aussi esthétiquement séduisante de la réussite économique. En outre, et dans le même ordre d'idée, Larry Ellison, grand adepte de la communication "spectaculaire » et de la médiatisation, participe à la Coupe de l'America (America's Cup), en association avec le constructeur automobile Allemand BMW ; son équipe (BMW Oracle Racing), sur son bateau trimaran (Oracle), remporte la coupe en février 2010.

\section{Acte 5 : L'ère de l'après-fordisme}

Enfin, la cinquième grappe technologique (technologies d'information et de communication, nouveaux matériaux, biotechnologies) est celle qui anime le cycle long contemporain, lequel repose également sur l'essor des fonctions directionnelles et financières (stimulées par la mondialisation), et sur les industries culturelles (stimulées par l'évolution des goûts des consommateurs). Ces technologies sont à l'origine de nombreux produits, comme les ordinateurs personnels, les téléphones portables ${ }^{29}$ ou les produits multimédias par exemple. En outre, en raison de leur caractère universel et transversal, les technologies d'information et de communication ont un potentiel considérable pour transformer les méthodes de travail dans la plupart des secteurs d'activité. Même si les industries de haute technologie connaissent bien souvent une croissance sectorielle soutenue, le cycle d'accumulation induit par ces technologies reste marqué par une croissance générale médiocre et au caractère erratique ${ }^{30}$. Par ailleurs, le cycle d'expansion contemporain est marqué par la mise en concurrence accrue des capitalismes nationaux via l'accélération de la mondialisation, par la flexibilisation des méthodes de production, mais aussi par la fragilisation de la classe salariale et par la remise en question de la régulation keynésienne héritée du fordisme. En réaction à la performance du modèle industriel japonais, les industries traditionnelles américaines adoptent depuis les années 1980 des stratégies innovantes d'organisation du travail, fondées sur la flexibilité. Phénomène particulièrement sensible dans des secteurs tels que l'automobile, la "firme-réseau" remplace la firme pyramidale aux relations plus clairement hiérarchiques de la période précédente (voir Lung 1991). Cependant, d'autres formes de flexibilisation apparaissent, via le recours à des stratégies de redistribution spatiale des établissements de production manufacturière, via le recours aux technologies innovantes (comme la robotisation), mais aussi via une refonte en profondeur du marché du travail. Au final, cette transformation radicale du marché du travail, qui débouche sur la remise en cause du rapport salarial fordien fondé sur le contrat à durée indéterminée (CDI), conduit au retour de la fragilité du salariat (Castel 1995).

Ce cinquième cycle long (1992/98- ?) correspond à l'ère de l'« après-fordisme $»^{31}$. Les dates bornant au départ ce cycle restent en débat ; 1992 correspond à la reprise de la croissance aux États-Unis, 1998 à la reprise de la croissance en Europe. Même si nous manquons encore de recul, les grandes lignes des structures spatiales de ce cycle peuvent être identifiées. Les industries motrices de ce cycle (haute technologie, finances, industries culturelles) permettent à certaines métropoles de la Sunbelt (Californie, Arizona, Colorado, Texas, Floride surtout) d'avancer sur leur trajectoire de 
développement. Ce sont par exemple Los Angeles avec l'industrie cinématographique articulée de plus en plus aux technologies numériques de traitement de l'image (Scott 2005), ou la région de San Francisco qui voit se développer les biotechnologies et désormais les "greentech" (voir Ktitareff 2009; Sengès 2009), ainsi que différentes composantes de l'économie culturelle ou de l'économie de la connaissance (Leriche et Rubin 2011). Ces industries motrices permettent également à la plupart des métropoles $\mathrm{du}$ cadran Nord-est du pays de réussir une reconversion économique parfois spectaculaire, à l'instar de Minneapolis (production d'ordinateurs), de Boston (équipement électronique et informatique), ou encore de Chicago (activités directionnelles et financières). Après des difficultés considérables au cours de la décennie 1970 (voir Castells 1976), l'économie new-yorkaise en particulier a trouvé un nouveau souffle grâce aux activités financières et directionnelles qui font de cette métropole le principal centre de commandement de l'économie mondiale (Sassen 1991). Dans ce nouveau paradigme économique centré sur les industries créatives et les industries de la connaissance, les universités et centres de recherche jouent un rôle prépondérant dans les dynamiques territoriales de l'industrie. Dans cette perspective, le Nord-est du pays reste, en dépit de la croissance de la Sunbelt, un pôle majeur à l'échelle du pays et même au-delà, tant la puissance scientifique, culturelle et universitaire, industrielle, financière et directionnelle du quadrant Nord-est du pays reste considérable.

La Sears Tower, localisée dans le Loop de Chicago, est emblématique de la puissance du quadrant Nord-est des États-Unis, à l'échelle du pays et sans doute à l'échelle globale (cf. figure 5) ${ }^{32}$. Inaugurée en 1974, cette tour est destinée initialement à regrouper en un même lieu les personnels du siège social de la société de commerce de détail Sears Roebuck \& Cie éparpillés en différents lieux de Chicago. Néanmoins, au-delà de cet impératif fonctionnel, la Sears Tower est aussi le fruit de la volonté de l'entreprise de mettre en évidence sa puissance sur la scène urbaine de Chicago, contribuant ainsi activement à la transformation de la skyline de la métropole. La Sears Tower reste le plus haut gratte-ciel du monde entre 1974 et 1998 ; dessinée par Bruce Graham (1925-2010) du cabinet Skidmore, Owings \& Merrill (SOM), la tour est aussi le symbole du savoirfaire de Chicago en matière d'architecture. Néanmoins, s'inscrivant dans le cadre du mouvement plus général de suburbanisation des fonctions directionnelles - qui concourt au développement des edge cities (Garreau 1991) -, la société Sears quitte le bâtiment au milieu des années 1990 pour s'installer en banlieue. Rachetée, la tour change de nom et devient officiellement la Willis Tower durant l'été 2009. Si cette course à la construction d'immeubles de grande hauteur (IGH) manifeste la volonté d'affirmer sa puissance de la part du capitalisme américain, il est à noter que cette volonté est aujourd'hui battue en brèche par le capitalisme de la Chine ou des pétromonarchies.

\section{Conclusion : mise en scène et trompe-l'œil}

Comme nous l'avons vu, la mobilité sectorielle et géographique du capital conduit à l'émergence continuelle de nouveaux espaces industriels, comme la Silicon Valley qui, depuis les années 1960, fait office de figure emblématique de la capacité de renouvellement du capitalisme américain (voir Saxenian 1994). Inversement, cette mobilité provoque l'obsolescence et l'abandon des anciens espaces industriels, à l'instar de quelques-unes des plus grandes métropoles et de nombreuses villes de l'Industrial 
Belt, du coup transformée en Rust Belt, comme Detroit, Pittsburgh ou Akron. Cependant, comme le montrent les exemples du Mill Building à Lowell, du Pabst Brewery Complex à Milwaukee ou du Ford Rouge Factory Visitor Center, il peut arriver que certains espaces soient réinsérés dans la machinerie économique. En de telles occasions, ces espaces changent d'usage, la fonction originelle et première - essentiellement manufacturière - des bâtiments cédant alors la place à une fonction plus idéelle et sémiotique (diffusion de la culture historique, éducation, divertissement).

Cette mobilité du capital et les répercussions de cette mobilité sur le développement régional sont à considérer comme une sorte de toile de fond (le décor de la pièce de théâtre), sur laquelle prend place la mise en scène urbaine du capital (le texte de la pièce) (voir Ryngaert 2000). Les fonctions de production (faire et savoir-faire d'un côté) et de communication (faire et faire savoir de l'autre) sont donc indissociables. Si les tâches banales, les tâches industrieuses, se traduisent par la genèse d'espaces plutôt fonctionnels (usines, entrepôts, etc.), les tâches directionnelles tendent à se traduire par la genèse d'espaces à forte charge symbolique (immobilier d'entreprise et bâtiments prestigieux, etc.). L'architecture est, dans cette perspective, un outil de communication particulièrement performant; la multiplication des immeubles de grande hauteur est la parfaite illustration de l'idée de Jonhson (1996) selon qui :

Dans le monde du commerce, le gratte-ciel est né parce nous n'avions pas d'autre religion à exprimer. Mais il s'agissait d'une volonté d'expression, et non pas d'une nécessité économique (7) ${ }^{33}$

Les Central Business District, qui à travers l'ensemble du territoire des États-Unis connaissent en conséquence un processus de "Manhattanization» (Brugmann et Sletteland 1971), sont les premiers bénéficiaires de cette mise en scène du capital.

Néanmoins, à l'autre bout du spectre sociogéographique, certains quartiers sont sévèrement touchés par le sous-emploi et la misère sociale; en d'autres termes, si le capital, de différentes manières que nous n'avons fait qu'effleurer, se met en scène dans l'espace urbain, laissant accroire que sa mobilité résulte d'un processus cyclique et heureux d'expansion géographique et de succès économique, la réalité mérite quelques nuances. L'exemple de l'opération Renaissance Center à Detroit illustre à l'envi cette contradiction inhérente au capitalisme, capable de créer conjointement des espaces symbolisant la réussite et la performance, et des espaces symbolisant l'abandon, l'obsolescence et la misère (cf. figure 6) ${ }^{34}$. Detroit illustre de manière sans doute caricaturale comment les stratégies du capital peuvent masquer des réalités peu glorieuses. Construit entre 1973 et 1977 sur des financements de General Motors (GM), Renaissance Center est un immense complexe immobilier constitué des sept gratte-ciel hébergeant essentiellement des bureaux - dont le siège social de GM - et un hôtel de luxe ${ }^{35}$. Censé incarner la "renaissance», justement, de Detroit, le complexe fait pourtant, dès les années 1980, figure de chant du cygne pour l'économie locale, à mesure que les constructeurs automobiles de Detroit subissent la concurrence des constructeurs européens et, surtout, nippons, et qu'ils déploient parallèlement des stratégies capitalistiques débouchant sur le rachat de sociétés à l'étranger et sur le sous-investissement dans l'outil productif domestique. Cette mise en scène du capital $\mathrm{du}$ secteur automobile américain, sous la forme d'un projet architectural et urbanistique ambitieux, moderne et spectaculaire à la Debord [1971], est la traduction d'une stratégie plus générale de communication et de marketing destinée à augmenter la notoriété, le prestige et, au final, le chiffre d'affaires et les profits des entreprises. Néanmoins, pour le cas spécifique de Detroit, cette mise en scène évoque finalement un 
décor de carton-pâte digne des studios hollywoodiens ; loin du Central Business District, les quartiers populaires s'enfoncent dans le chômage et la misère (voir Staszack 1999). Les difficultés économiques et sociales de Detroit depuis les années 1980 sont telles que la métropole en arrive aujourd'hui à incarner l'envers du « rêve américain » (Popelard et Vannier 2010).

\section{BIBLIOGRAPHIE}

Aglietta, Michel. Régulation et crises du capitalisme. 1976. Paris: O. Jacob, 1997.

Arnold, Erik et Guy, Ken. « Actions possibles pour promouvoir la croissance grâce aux technologies de l'information. » In Technologies de l'information et les nouveaux domaines de croissance. Ed. OCDE. Paris, 1989. 145-221.

Bergeron, Louis et Maiullari, Maria Teresa. « Le gigantisme industriel, de la guerre de Sécession à la grande crise. » La Revue (des Arts et Métiers) 19 (1997).

Bluestone, Barry and Harrison, Bennett The Desindustrialization of America. New York : Basic Books, 1982.

Boyer, Robert. La théorie de la régulation : une analyse critique. Paris : La découverte, 1986.

---. Une théorie du capitalisme est-elle possible ? Paris: O. Jacob, 2004.

Brugman, Bruce and Sletteland, Greggar (ed.). The Ultimate Highrise: San Francisco's Mad Rush Toward the Sky. San Francisco : San Francisco Bay Guardian Books, 1971.

Castel, Robert. Les Métamorphoses de la question sociale, une chronique du salariat. Paris : Fayard, 1995.

Castells, Manuel. « La crise urbaine aux États-Unis : vers la barbarie ? » In Les Temps Modernes 355 (1976): 1177-1240.

---. The Informational: City Information Technology, Economic Restructuring, and the Urban-regional Process. Cambridge: Basil Blackwell, 1989.

Cochran, Thomas. The Pabst Brewing Company: History of an American Business. Milwaukee: BeerBooks.com, 2006.

Davis, Mike. City of Quartz. Los Angeles, capitale du futur. Paris : La découverte, 1997.

Debord, Guy. La Société du spectacle. 1971. Paris : Ed. Gérard Lebovici, 1989.

DeMarco, Gordon. A Short History of Los Angeles, San Francisco: Lexikos, 1988.

Dupré, Judith. Gratte-ciel du monde : une histoire des plus célèbres gratte-ciel du monde. New York: Köln Black Dog \& Leventhal Publishers/Könemann Verlag, 1996.

Fields, Garry. Territories of Profit: Communications, Capitalist Development, and the Innovative Enterprises of G. F. Swift and Dell Computer. Stanford: Stanford University Press, 2004.

Garreau, Joel Edge City: Life on the New Frontier. New York : Doubleday, 1991.

Gimpel, Jean La Révolution industrielle du Moyen Age. Paris: Seuil, 1975. 
Hall, Peter. "The Geography of the Fifth Kondratieff." In Silicon Landscapes. Ed. Hall, Peter and Markusen, Ann, Boston: Allen \& Unwin, 1985. 1-19.

Harvey, David. The Urbanization of Capital. Baltimore: Johns Hopkins University Press, 1985.

---. Spaces of Capital: Towards a Critical Geography. New York: Routledge, 2001.

---. Limits to Capital. London: Verso, 2007.

Kondratieff, Nikolai D. « The Long Waves in Economic Life. » 1925. Review of Economic Statistics 17 : 6 (1936) : 105-115.

Krugman, Paul et Obsfeld, Maurice Économie internationale. Bruxelles : De Boeck Université, 1995.

Ktitareff, Michel. Révolution verte : enquête dans la Silicon Valley. Paris : Dunod, 2009.

Leriche, Frédéric. « Immigration et question identitaire aux États-Unis ». In Géopolitiques des Amériques. Ed. Thébault, Vincent et al. Paris : Nathan, 2009. 285-301.

Leriche, Frédéric et Rubin, Jasper. « Economie culturelle et urbanisation : San Francisco, produit culturel. » In Regards croisés sur San Francisco. Ed. Christol, Hélène et al. Aix-en-Provence : Presses Universitaires de Provence, 2011. A paraître.

Leriche, Frédéric et Scott, Allen. « Hollywood, un siècle d'industrie cinématographique.» In L'Economie culturelle et ses territoires. Ed. Leriche, Frédéric et al. Toulouse : Presses Universitaires du Mirail, 2008. 29-41.

Lipietz, Alain. « Le national et le régional : quelle autonomie face à la crise capitaliste mondiale? ». In La dynamique spatiale de l'économie contemporaine. Ed. Benko, Georges, La GarenneColombes : Editions de l'Espace Européen, 1990. 71-103.

Lung, Yannick. Les Métamorphoses de la Ford Motor Company : de l'entreprise pyramidale à la firmeréseaux? Bordeaux : IERSO-Université Bordeaux 1, 1991.

Maddison, Angus. L'Economie mondiale, une perspective millénaire. Paris : OCDE, 2001.

Mangin, Claude. « Géographies d'une ville industrielle américaine : Lowell en modèles chorématiques. » Mappemonde 68:4 (2002): 25-29.

Markusen, Ann. Profit Cycles, Oligopoly, and Regional Development. Cambridge, Mass.: MIT Press, 1985.

Markusen, Ann and Hall, Peter (eds.). The rise of the Gunbelt: the Military Remapping of Industrial America. New York: Oxford University Press, 1991.

Marx, Karl. Le Capital. 1867. Paris: PUF, 1993.

Messine, Philippe. Les saturniens. Quand les patrons réinventent la société. Paris: La découverte, 1987.

Norton, R.D. and Rees, John. « The Profit Cycle and the Spatial Decentralization of American Manufacturing. » Regional Studies 13 (1979) : 141-151.

Popelard, Allan et Vannier, Paul. « Detroit, la ville afro-américaine qui rétrécit. » Le Monde diplomatique 670 (2010) : 10-11.

Ryngaert, Jean-Pierre. Introduction à l'analyse du théâtre. Paris: Nathan, 2000.

Sassen, Saskia. The Global City: New York, London, Tokyo. Princeton: Princeton University Press, 1991.

Saxenian, AnnaLee. Regional Advantage: Culture and Competition in Silicon Valley and Route 128. Cambridge, Mass.: Harvard University Press, 1994. 
Schumpeter, Joseph. Business Cycles. New York: McGraw-Hill, 1939.

---. Capitalisme, socialisme et démocratie. 1942. Paris : Payot, 1974.

Scott, Allen. « The Aerospace-electronics Industrial Complex of Southern California: the Formative Years, 1940-1960. » Research Policy 20:5 (1991): 439-456.

---. On Hollywood: the Place, the Industry. Princeton: Princeton University Press, 2005.

Scott, Allen and Angel, David. « The Global Assembly Operations of US Semiconductor Firms: a Geographical Analysis » Environment and Planning A 19 (1987): 875-912.

Scott, Allen and Paul, Allan. «Industrial Development in Southern California: 1970-1987 ». In Our Changing Cities. Ed. Hart J. Baltimore: Johns Hopkins University Press, 1991. 189-217.

Sengès, Anne. Eco-tech : moteurs de la croissance verte en Californie et en France. Paris : Autrement, 2009.

Smith, Adam. Recherches sur la nature et les causes de la richesse des nations. 1776. Paris: Folio, 1976.

Soja, Edward, Morales, Rebecca and Wolff, Goetz. « Urban Restructuring: an Analysis of Social and Spatial Change in Los Angeles » Economic geography 59 (1983): 198-230.

Staszack, Jean-François. "Détruire Detroit. La crise urbaine comme produit culturel” Annales de Géographie 607 (1999) : 277-299.

Storper, Michael and Walker, Richard. The Capitalist Imperative. New York: Basil Blackwell, 1989.

Walker Richard. « Another round of globalization in San Francisco. » In Urban Geography 17/1 (1996): 60-94.

\section{NOTES}

1. Joseph Schumpeter (1883-1950), économiste autrichien; théoricien de l'innovation technologique, des cycles économiques et du développement capitaliste, il avance la notion de destruction créatrice, qu'il considère comme un moteur de l'expansion économique.

2. David Harvey est l'un des plus célèbres géographes Américains contemporains ; il est connu en particulier pour ses analyses critiques des dynamiques du capitalisme.

3. G.F. Swift est l'entreprise pionnière et leader en matière d'industrialisation du conditionnement de la viande de bœuf à la fin du XIX ${ }^{\text {ème }}$ siècle; Dell est une entreprise majeure du marché contemporain des micro-ordinateurs, spécialisée dans la production d'ordinateurs spécialisés et adaptés au client.

4. L'« école de la régulation» est une école hétérodoxe de la recherche en économie. D'inspiration néo-marxiste, l'approche épistémologique et méthodologique de cette école repose sur la prise en considération de la réalité concrète des faits économiques. École extrêmement influente à travers le monde, elle se divise en deux courants; un courant "parisien » (représenté en particulier par Michel Aglietta, Robert Boyer ou Alain Lipietz) et un courant "«grenoblois " (représenté essentiellement par Gérard Destanne de Bernis). Après avoir étudié les transformations du capitalisme dans le temps, l'école de la régulation a étudié la variété des formes du capitalisme dans l'espace. 
5. Économiste Russe, directeur de l'Institut de conjoncture de Moscou, Nikolaï Kondratieff "découvre» dans les années 1920 que les économies capitalistes se développent selon un rythme cyclique d'environ 50 années. Il publie un article (en russe) en 1925, traduit en allemand en 1926, puis en anglais en 1936 ("The long waves in economic life "; le texte n'est pas disponible en français). La signification de cette thèse est claire : le capitalisme est doté de capacités internes de rebond, et après chaque crise structurelle, il connaît une nouvelle phase d'expansion. C'est donc reconnaître la vitalité intrinsèque du capitalisme, sa pérennité potentielle, malgré les crises. Cette thèse, pour un régime soviétique qui parie sur la chute finale du capitalisme, est jugée comme étant hérétique, et provoque une profonde discorde entre Kondratieff et Trotski en particulier. En 1930, Kondratieff est déporté dans un goulag, si bien que si sa date de naissance est connue (1892), la date de son décès est incertaine (1930 ou 1938?).

6. Voir aussi, sur ce point, l'ouvrage Business Cycles (1939), non disponible en français.

7. Michael Storper et Richard Walker, géographes Américains, sont parmi les plus éminents spécialistes de géographie économique dans le monde. Ils appartiennent à ce qu'il est convenu d'appeler l'"école de géographie de Californie".

8. Si les travaux théoriques de David Harvey sont essentiels sur cette question, l'analyse plus empirique que propose Philippe Messine (1987) de la stratégie spatiale du capital (General Motors en l'occurrence) pour tenter de résoudre ses difficultés en créant une nouvelle marque automobile (Saturn) dans un nouvel espace (le Tennessee) est remarquable. Cette stratégie est l'occasion d'une remise à plat en matière de relations entre le patronat et les syndicats (clef de voûte du projet), mais aussi, consécutivement, en matière de positionnement commercial, de technologie industrielle (la robotisation), de méthodes d'organisation de la production.

9. Qui plus est, l'existence même de ces cycles continue à être débattue par certains économistes.

10. Sur le déplacement géographique et historique des centres d'impulsion de l'économie, voir Angus Maddison (2001) qui étudie cette question à l'échelle spatiale mondiale et à l'échelle temporelle du millénaire.

11. "Première" entre guillemets, puisque les historiens médiévistes ont mis en évidence qu'une véritable révolution industrielle s'est produite au Moyen Age. Comme l'explique Jean Gimpel (1975): «La première » révolution industrielle date du Moyen Age. Les XI ${ }^{\text {ème}}$, XII ${ }^{\text {ème }}$ et XIII ${ }^{\text {ème }}$ siècles ont créé une technologie sur laquelle la révolution industrielle $\mathrm{du} 18^{\mathrm{ème}}$ siècle s'est appuyée pour prendre son essor. (...) La société médiévale remplaça le travail manuel, souvent forcé des esclaves, par le travail des machines. (9) Une fois cette nuance établie, il convient de préciser que ladite (par convention) " première » révolution industrielle est généralement considérée comme la Révolution Industrielle (c'est nous qui soulignons).

12. Cette forme de désintégration du processus productif renvoie à la notion de «division sociale du travail » entre les firmes, mise en avant initialement par Adam Smith [1776].

13. Les économies d'échelle (ou rendements croissants) peuvent être compris comme une baisse des coûts de production-associée à l'amélioration de la productivité-par unité de bien ou service produite, à mesure que s'allongent les séries de ces biens et services produits. Autrement dit, il y a des économies d'échelle quand le doublement (par exemple) des intrants dans une entreprise (on parle dans ce premier cas 
d'économies d'échelle internes) ou une industrie (on parle dans ce second cas d'économies d'échelle externes) accroit la production finale d'un facteur supérieur à deux.

14. Figure 1. Source: Wikipedia. < http://fr.wikipedia.org/wiki/ Fichier:Mill_Building_\%28now_museum\%29,_Lowell,_Massachusetts.JPG> consulté le 18/03/11.

15. Les économistes de l'école de la régulation ont peu (voire pas) travaillé sur la période antérieure à 1848 .

16. Henry Bessemer (1813-1898), ingénieur Anglais, invente un procédé innovant qui révolutionne la technique de production de l'acier (brevet déposé en 1856).

17. Figure 2. Source: Wikipedia. < http://en.wikipedia.org/wiki/ File:Former_brewery_Pabst_Brewing_Company_in_Milwaukee_Wisconsin.jpg> consulté le 18/03/11.

18. Sur le site Internet de la société de promotion immobilière, le promoteur - Joseph Zilber - est présenté comme un philanthrope amoureux de la ville où il a grandi. $<$ http://www.thebrewerymke.com/index.htm> consulté le 18/03/11.

19. C'est en particulier le cas de l'immense usine créée par Ford à Dearborn en 1908.

20. Les investissements américains à l'étranger ont cependant commencé bien plus tôt, dès la fin du XIXème siècle en Amérique latine (principalement pour accéder aux ressources primaires du continent). Dans les premières décennies du XX⿳亠丷⿵冂丶 ${ }^{\text {èe }}$ siècle pour accéder aux marchés, les industries de masse émettent des IDE, à l'instar de Ford ou de General Motors (qui rachète le producteur Allemand Opel en 1926).

21. Wilmington, DE est le berceau de l'entreprise Du Pont de Nemours.

22. La stratégie de développement de l'agriculture irriguée est aussi (surtout?), pour la Californie du Sud, une stratégie de développement destinée à tirer profit de la rente foncière via une promotion immobilière adossée à une véritable campagne publicitaire qui diffuse dès le dernier quart du XIX ${ }^{\text {ème }}$ siècle le slogan «Oranges for health, California for wealth». Comme le rappelle Gordon DeMarco (1988) : «The citrus industry made sure the message was taken to all parts of the nation" (49). Sur cette stratégie (le boosterism, terme intraduisible en français) destinée à développer la Californie du Sud, voir aussi Mike Davis (1997).

23. Figure 3. Source : Center for Land Use Interpretation (CLUI). <http://www.clui.org/lotl/v29/d.html> consulté le 18/03/11.

24. Source: Brand Cultural Entertainment Media \& Events (BRC). < http:// www.brcweb.com/brand/fordRouge.html> consulté le 18/03/11.

25. Cette fermeture relative se mesure par la part du commerce extérieur dans le PIB. Pour la plupart des pays industriels, cette part atteint son plus bas niveau depuis la fin du XIX ${ }^{\text {ème }}$ siècle au milieu des années 1960. Comme le souligne Alain Lipietz (1990) :

[C]'est vers 1965 que le rapport entre les exportations et le marché intérieur, dans la plupart des pays capitalistes industrialisés, atteint son point bas historique. (85)

Les systèmes productifs nationaux sont alors à un degré de cohérence économique maximum.

26. Sur l'impact des dépenses d'armement dans la reconfiguration de la géographie économique des Etats-Unis, voir les travaux sur la « Gunbelt » de Ann Markusen et Peter Hall (1991). 
27. Pour la Californie du Sud : sur l'histoire de l'industrie sidérurgique, voir Mike Davis (1997) ; sur la reconversion industrielle, voir Allen Scott et Allan Paul (1991).

28. Figure 4. Source: Wikipedia. < http://en.wikipedia.org/wiki/ File:Oracle_Headquarters_Redwood_Shores.jpg> consulté le 18/03/11.

29. L'Europe, via la société Nokia en particulier, a cependant joué un rôle clef dans le développement de ce type de technologies et de marchés.

30. En ce sens, la croissance contemporaine semble renouer avec les modalités de la croissance de la fin du XIX ${ }^{\text {ème }}$ siècle.

31. Définir un objet scientifique par ce qu'il n'est pas est assez dérangeant, et ce terme de l'après-fordisme est peu satisfaisant. Faute de terme approprié, nous l'utilisons tout de même.

32. Figure 5. Source : Wikipedia.

<http://fr.wikipedia.org/wiki/Fichier:Sears_Tower_ss.jpg> consulté le 18/03/11.

33. Voir l'entretien que Philip Johnson a accordé à Judith Dupré (1996).

34. Figure 6. Source : Wikipedia.

<http://fr.wikipedia.org/wiki/Fichier:GM_headquarters_in_Detroit.JPG> consulté le 18/03/11.

35. L'architecte en est John Portman, du cabinet SOM.

\section{RÉSUMÉS}

Les États-Unis sont caractérisés par une très grande mobilité spatiale des facteurs de production, à savoir le capital et le travail. Reposant sur une analyse géographique, cet article décrit comment la mobilité géographique du capital (considéré ici comme un élément moteur de la transformation des espaces américains) se traduit dans un processus de consommation/ production d'espace. L'ambition d'une telle analyse est de comprendre comment la mobilité du capital se met en scène dans les espaces urbains, lieux par excellence de la concentration et de l'amalgamation du capital et du travail. Le «drame » de la mobilité spatiale du capital et de sa mise en scène, à l'échelle de l'histoire des États-Unis, se joue en cinq "actes ", analysés successivement à partir de trois éclairages différents (moteur technologique et industriel, inscription spatiale, mise en scène paysagère du capitalisme). L'article commence néanmoins par expliciter le cadre théorique dans lequel s'inscrit cette lecture de la mobilité du capital.

Intensive mobility of the factors of production (capital and work) is a major feature of the United States. Based on a geographical analysis, this paper describes the way in which the geographical mobility of capital (considered here as a driving force behind the reshaping of the American landscape) generates a process of both consumption and production of space. This approach aims at understanding how the mobility of capital is staged in urban spaces; such urban spaces are, by excellence, places of concentration and amalgamation of capital and work. The "drama » of the spatial mobility of capital and of its staging, which has unfolded throughout the history of the United States, falls into five " acts ». The present article will analyse these five acts from three different angles: from the perspective of technological and industrial engines, of spatial 
inscription, and eventually from that of the visual staging of capitalism. Nevertheless, the paper will begin with an explanation underlying our understanding of the spatial mobility of capital.

INDEX

Mots-clés : capitalisme, technologie, industrialisation, métropoles, architecture

Keywords : capitalism, technology, industrialization, metropolises, architecture

\section{AUTEURS}

\section{FRÉDÉRIC LERICHE}

Professeur

Université de Versailles Saint-Quentin-en-Yvelines

frederic.leriche@uvsq.fr 